\title{
A retrospective study on 226 polycythemia vera patients: impact of median hematocrit value on clinical outcomes and survival improvement with anti-thrombotic prophylaxis and non-alkylating drugs
}

\author{
Elena Crisà • Ermanno Venturino $\cdot$ Roberto Passera $\cdot$ Marco Prina • \\ Piercarla Schinco • Alessandra Borchiellini • Valentina Giai • \\ Maria Ausilia Ciocca Vasino • Mario Bazzan • Antonella Vaccarino • \\ Mario Boccadoro • Dario Ferrero
}

Received: 19 September 2009 / Accepted: 28 December 2009/Published online: 10 February 2010

(C) Springer-Verlag 2010

\begin{abstract}
The clinical impact of polycythemia vera (PV) diagnostic and therapeutic guidelines is still undetermined. In particular, the recommended target of hematocrit (Hct) $<0.45$ has been recently questioned and alkylating drugs are still used for elderly patients. We revised, according to WHO criteria, $300 \mathrm{PV}$ diagnosis and evaluated the impact
\end{abstract}

E. Crisà $\cdot$ P. Schinco $\cdot$ A. Borchiellini $\cdot$ V. Giai $\cdot$ M. Boccadoro $\cdot$

D. Ferrero $(\square)$

Divisione di Ematologia dell' Università di Torino,

Azienda Ospedaliera S. Giovanni Battista di Torino,

via Genova 3,

10126 Turin, Italy

e-mail: dario.ferrero@unito.it

E. Venturino $\cdot$ M. Prina

S.I.M.T. Banca del Sangue,

Azienda Ospedaliera S. Giovanni Battista di Torino, corso Bramante, 88 ,

10126 Turin, Italy

R. Passera

Medicina Nucleare II,

Azienda Ospedaliera S. Giovanni Battista di Torino,

corso Bramante, 88 ,

10126 Turin, Italy

M. A. Ciocca Vasino

Divisione Ospedaliera di Ematologia,

Azienda Ospedaliera S. Giovanni Battista di Torino, corso Bramante, 88,

10126 Turin, Italy

M. Bazzan · A. Vaccarino

Ambulatorio di Ematologia,

Casa di Cura "Cellini”,

via Cellini, 5 ,

10126 Turin, Italy on clinical outcome of median Hct and of the strategy to administer anti-thrombotic prophylaxis and to avoid alkylating chemotherapy in almost all patients. Of 226 patients with WHO-confirmed diagnosis (median age 66), 91.3\% survived at the median follow-up of 5.84 years and $77.5 \%$ are projected alive at 13 years. Eighteen percent had major thrombosis and $2.7 \%$ acute myeloid leukemia. Twenty-two percent of patients maintained an Hct $<0.45$ : their overall and thrombosis-free survival are similar to those of patients with a $0.45-0.48$ value. Conversely, an Hct $>0.48$ and a "high thrombotic risk" according to ECLAP criteria were both significantly associated to shorter survival and higher thrombosis risk. Chemotherapy reduced thrombotic events without affecting survival. Our study revealed suboptimal compliance to published guidelines. However, in our casistic characterized by wide use of anti-platelet- and avoidance of alkylating drugs, patients' survival, although analyzed retrospectively, seemed to have improved compared to old literature data. The optimal Hct target was not clearly defined, although a value $<0.48$ looks highly advisable.

Keywords Polycythemia vera - Thrombotic events . Chemotherapy · Hematocrit · Acute myeloid leukemia

\section{Introduction}

Polycythemia vera (PV) is the most common chronic myeloproliferative neoplasm, with an annual incidence of 2.3 per $100,000[1,2]$, a reported median survival of 10 15 years [3-6] and an overall mortality of 3.5 per 100 person/year [5]. Thrombotic complications are the main 
cause of morbidity and mortality, occurring in more than one third of patients and causing $35-45 \%$ of deaths $[5,6]$. Other possible adverse events are the evolution into secondary myelofibrosis (MF) or acute myeloid leukemia (AML) occurring in 10-15\% [4, 6-8] and 2-15\% [4, 8-16] of patients, respectively, depending on treatments and with increased incidence with long-standing disease.

Diagnostic criteria have been revised many times since their first publication by the Polycythemia Vera Study Group in 1975 [17, 18]. Recently, the World Health Organization (WHO) proposed new criteria, in consideration of the discovery of JAK2 mutation role in PV [19, 20]. However, before the discovery of JAK2 mutations [21-25], differential diagnosis between PV and secondary erythrocytosis was not always immediate and it is unclear how previous WHO diagnostic recommendations have been actually followed in general practice, outside of clinical trials.

Current therapy guidelines recommend phlebotomy as firstline treatment, in order to maintain the hematocrit (Hct) value below 0.45 and prevent cardiovascular accidents [18]. Lowdose acetyl-salicylic acid (ASA) has been recommended too, unless contraindicated, in order to reduce thrombotic risk and mortality; conversely cytoreductive therapy is recommended only in the presence of poor tolerance to phlebotomy, symptomatic or progressive splenomegaly or evidence of high thrombotic risk or disease progression [18, 26-29].

In spite of well-established therapeutic guidelines, some doubts still remain about the optimal management of PV patients. Indeed, a recent European survey on a very large casistics (ECLAP study) questioned the actual role of a low $(<0.45)$ Hct value in reducing the thrombotic risk [29, 30]. Moreover, the same study evidenced that even in the setting of a clinical study, about $60 \%$ of patients did not reach or maintain the recommended Hct value [30]. Therefore, it is unclear how the strict requirement of intensive Hct reduction has been actually satisfied outside of clinical trials. For chemotherapy-requiring patients, hydroxyurea (HU) is generally recommended as first-line agent because of its unproven leukemogenicity [28]. However some alkylating agents like pipobroman and busulfan are still used, particularly in elderly patients [26].

In this study, we performed a retrospective analysis on 300 patients with a PV diagnosis, referred to a single blood bank for phlebotomy. The primary objectives of the study were: (1) to evaluate in our casistics the controversial role of Hct value in survival and thrombosis prevention; (2) to verify whether the strategy for near all patients of anti-thrombotic prophylaxis and avoidance of alkylating chemotherapy might have improved survival and reduced AML and major thrombosis incidence, compared to literature data. As secondary objectives, we verified in our patients the actual adherence to published diagnostic and therapeutic guidelines in routine management of PV and the prognostic role of some known risk factors.

\section{Methods}

The present analysis is a retrospective study based on the database of polycythemic patients of S. Giovanni Battista Hospital transfusion center in Turin, Italy. Patients were followed in three different hematological institutions but all of them referred to this single transfusion center for phlebotomy. Patients' data were cumulatively evaluated anonymously, in agreement to local ethical rules. Only patients with a diagnosis of PV made from January 1, 1995 to March 31, 2008 were included. Each diagnosis was revised to include in the study only the cases that fully satisfied either 2001 or 2007 WHO diagnostic criteria for PV. Data files were reviewed before inclusion in the present analysis and the times of observation were censored on December 31, 2008.

Primary end-points were overall survival (OS), defined as the time from diagnosis to death for any cause or last follow-up alive, time to occurrence of total thrombosis (TTT) and time to occurrence of major thrombosis (TMT). Major thrombotic events comprehended ischemic stroke, acute myocardial infarction, deep venous thrombosis and pulmonary embolism. Total thrombosis included all major events plus transient ischemic attacks, peripheral arterial thrombosis and superficial thrombophlebitis. Secondary end-points included the incidence and time to development of AML, solid tumors or secondary MF.

For each patient, the following parameters at diagnosis were evaluated: age; presence of cardiovascular risk factors such us hypertension, diabetes, cigarette smoking, dyslipidemia; history of thrombosis or bleeding. These parameters were used to define the thrombotic risk according to the recent definition by Finazzi and Barbui based on ECLAP data $[28,29]$ : low risk if younger than 60 and without prior thrombosis and cardiovascular risk factors; intermediate risk if younger than 60 , without history of thrombosis but with cardiovascular risk factors; high risk if aged more than 60 or with history of thrombosis.

During the follow-up, data regarding thrombotic events, bleeding, cytoreductive treatments, anti-platelet or anticoagulant therapy and laboratory values were recorded. Median values of Hct, platelets and leucocytes during the observational time (with the exclusion of blood counts performed after occurrence of MF, AML, or other neoplasms) were used to estimate their impact on survival, TTT, TMT, and time to hematological transformation: these data were evaluated on 211 patients ( $93 \%$ of total) who had at least three complete blood counts performed per year (average 5/year). The prognostic role of Hct value was analyzed with two cutoffs: 0.45 , corresponding to WHO recommendation, and 0.48 , a value in the upper normal range. Higher cutoff values (0.49 or more) were not considered, due to the very low number of patients $(<10 \%)$ maintained at so high Hct values. Cutoff values 
Table 1 Patients' general features are illustrated. The 3 groups of patients divided according to median Hct values include 211 patients only, who had at least 3 blood count/year of follow-up

Main clinical features of 226 patients with PV

\begin{tabular}{lcccccccc}
\hline & \multicolumn{2}{c}{ All the patients } & \multicolumn{2}{c}{ Median HCT $<0.45$} & \multicolumn{2}{c}{ Median HCT $0.45-0.48$} & \multicolumn{2}{c}{ Median HCT $>0.48$} \\
& No & $\%$ & No & $\%$ & No & $\%$ & No & $\%$ \\
No & 226 & & 46 & 22 & 131 & 62 & 34 \\
Males & 124 & 54.9 & 10 & 21.7 & 79 & 60.3 & 26 \\
Females & 102 & 45.1 & 36 & 78.3 & 52 & 39.7 & 8 & 23.6
\end{tabular}

Age at diagnosis-years (range)

$\begin{array}{lrrrrrrr}\text { Median } & 66(18-92) & 67(25-87) & & 64(18-83) & & 69(31-87) \\ \text { over 65 } & 117 & 51.8 & 28 & 60.9 & 61 & 46.6 & 20 \\ \text { under 65 } & 109 & 48.2 & 18 & 39.1 & 70 & 53.4 & 14\end{array}$

JAK2 V216F mutated

Positive

Negative

not determined

$\begin{array}{rrrrr}102 & 93.6^{\mathrm{a}} & 24 & 96.0^{\mathrm{a}} & 63 \\ 7 & 6.4^{\mathrm{a}} & 1 & 4.0^{\mathrm{a}} & 5 \\ 117 & 51.8 & 21 & 45.7 & 63\end{array}$

$92.6^{\mathrm{a}}$

$7.4^{\mathrm{a}}$

48.1

$\begin{array}{rr}10 & 90.9^{\mathrm{a}} \\ 1 & 9.1^{\mathrm{a}} \\ 23 & 67.6\end{array}$

Thrombotic risk ${ }^{\mathrm{b}}$

Low

$39 \quad 17.3$

13

28.3

18

13.7

18.3

Intermediate

$\begin{array}{ll}29 & 12.8\end{array}$

2.2

$\begin{array}{llll}158 & 69.9 & 32 & 69.6\end{array}$

High

$137 \quad 60.6$

54.3

Cardivascular risk factors

$55 \quad 24.3$

21.7

Prior major thrombosis

$\begin{array}{lll}34 & 15.0 & 7\end{array}$

Median follow-up-years (range)

$5.83(0.76-13.78)$

$\begin{array}{lll}151 & 66.8 & 31\end{array}$

15.2

Cytoreductive treatment

Hydroxyurea

$147 \quad 97.4^{\mathrm{c}} \quad 29$

Alkylants

Alpha interferon

Anti-platelet drugs

Anticoagulants drugs

$\begin{array}{rr}24 & 15.9^{\mathrm{c}} \\ 2 & 1.3^{\mathrm{c}} \\ 209 & 87.2 \\ 34 & 13.3\end{array}$

67.4

$93.6^{\mathrm{c}}$

$16.1^{\mathrm{c}}$

$0.0^{\mathrm{c}}$

93.5

13.0

24

89

67.9

64.1

25.2

16.0

21

6.60

85
85
11
2
123
20

64.9

$100^{\mathrm{c}}$

$12.9^{\mathrm{c}}$

$2.36^{\mathrm{c}}$

93.9

15.3

$\begin{array}{cc}2 & 100 \\ 0 & 0.0 \\ 0 & 0.0 \\ 0 & 0.0 \\ 0 & 0.0\end{array}$

24.0
32.0
24.0
16.0
4.0

3
3
4
3
0

23.1
23.1
30.8
23.1
0.0

11.1

44.4

22.2

Tumors (except AML)

Others

Unknown

Events rate (Patients/events)

\begin{tabular}{lrrrr} 
Rate of death & $25 / 226(11.1 \%)$ & $2 / 46(4.3 \%)$ & $13 / 131(9.9 \%)$ & $9 / 34(26.5 \%)$ \\
Rate total thrombosis & $58 / 226(25.7 \%)$ & $12 / 46(26.0 \%)$ & $30 / 131(22.9 \%)$ & $12 / 34(35.3 \%)$ \\
Rate of major thrombosis & $43 / 226(19.0 \%)$ & $8 / 46(17.4 \%)$ & $21 / 131(16.0 \%)$ & $11 / 34(32.4 \%)$ \\
Rate of tumors (AML excluded) & $15 / 226(6.6 \%)$ & $1 / 46(2.2 \%)$ & $10 / 131(7.6 \%)$ & $3 / 34(8.8 \%)$ \\
Rate of AML & $6 / 226(2.7 \%)$ & $1 / 46(2.2 \%)$ & $4 / 131(3.0 \%)$ & $1 / 34(2.9 \%)$ \\
Rate of MF & $11 / 226(4.9 \%)$ & $2 / 46(4.3 \%)$ & $5 / 131(3.8 \%)$ & $4 / 34(11.8 \%)$ \\
\hline
\end{tabular}

${ }^{\mathrm{a}}$ Of tested cases

${ }^{\mathrm{b}}$ According to Finazzi and Barbui ${ }^{(32,33)}$

${ }^{\mathrm{c}}$ Percent of chemotherapy-treated patients; some patients received more than one treatment 


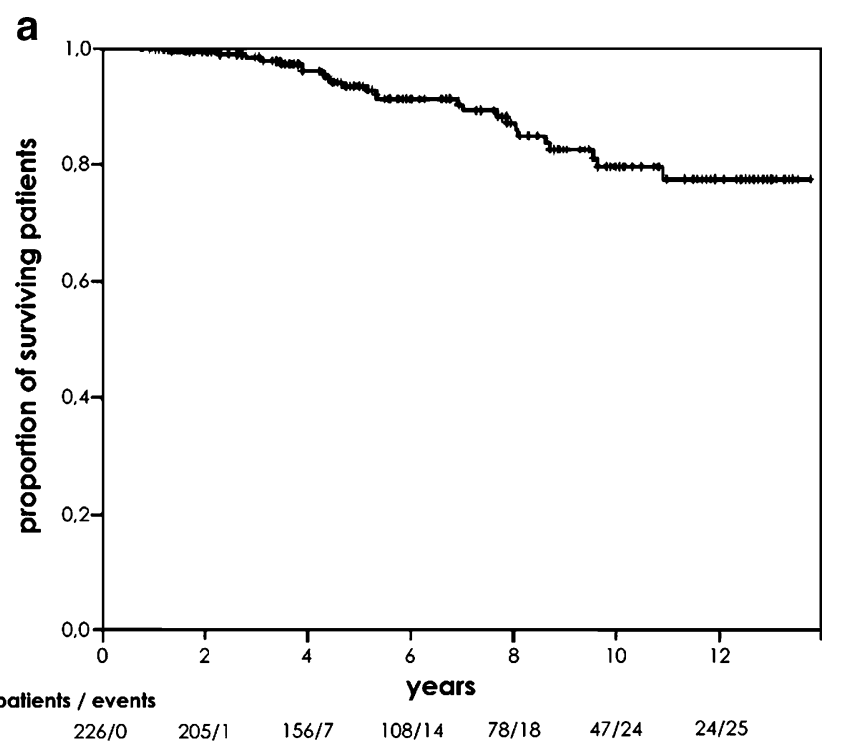

b
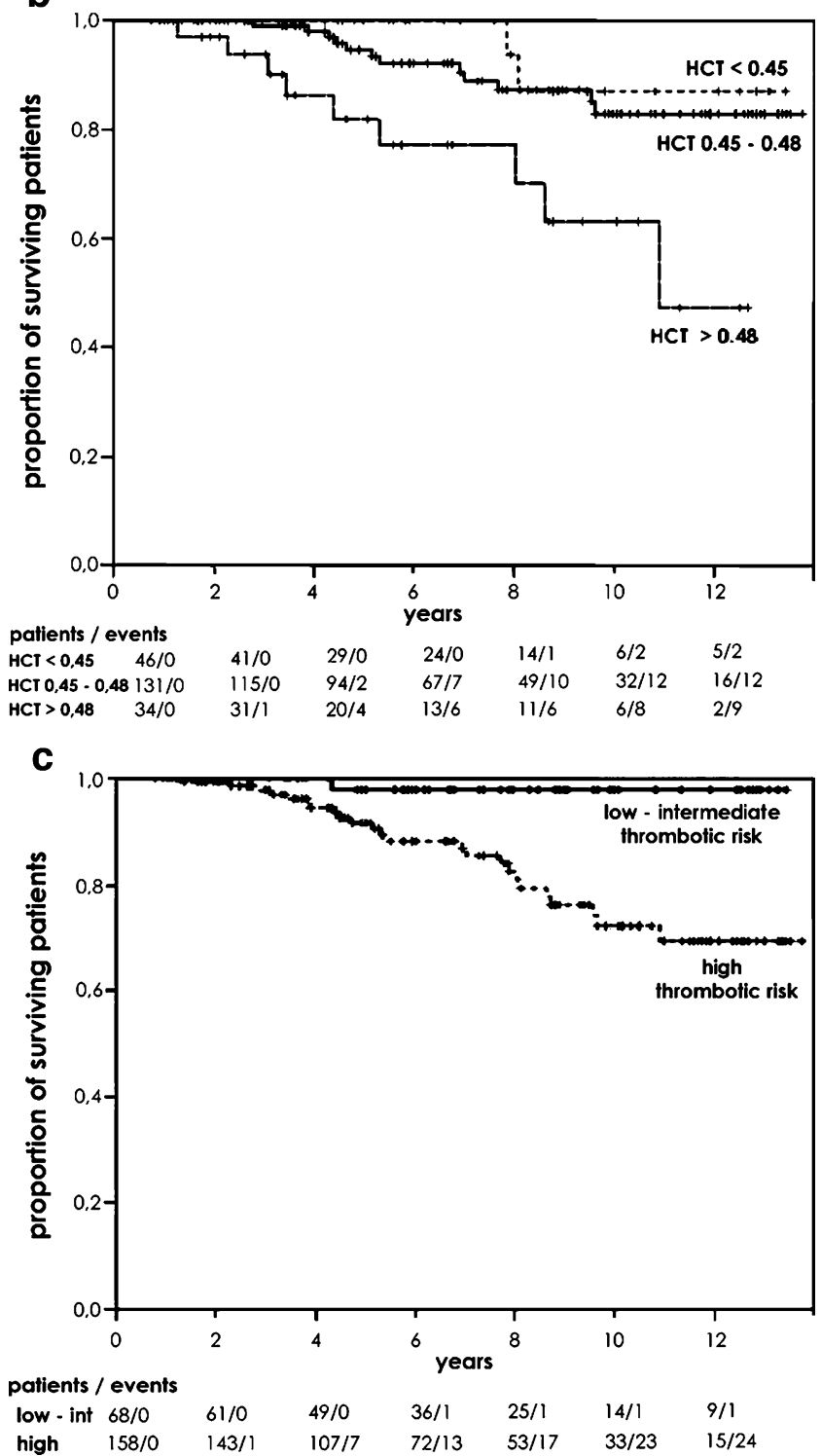

Springer
Fig. 1 Overall survival of 226 patients with polycythemia vera. a Overall survival: all patients. b Overall survival for median hematocrit, under 0.45 vs. $0.45-0.48$ vs. over 0.48 ( $p=0.001)$. c Overall survival for thrombotic risk according to Finazzi and Barbui [28, 29], high vs. low-intermediate $(p=0.038)$

for platelet counts were $400 \times 10^{9} / 1$, corresponding to the normal upper range, and $600 \times 10^{9} / 1$, the thrombocytosis threshold previously used by WHO guidelines for the diagnosis of essential thrombocythemia [31]. A leukocyte cutoff value of $15 \times 10^{9} / 1$ was chosen, since it has been previously reported to be prognostically relevant in terms of thrombosis-free survival [32].

Statistical methods

Patients' characteristics were compared using the Pearson $\chi^{2}$ test for discrete variables and the Mann-Whitney test for continuous variables. OS, TTT, TMT, time to development of AML, solid tumors or secondary MF were analyzed by the Kaplan-Meier method, comparing the two arms by the log-rank test and calculating $95 \%$ confidence intervals (CIs). Univariate and multivariate analyses were performed using the Cox model and the following variables were considered: gender (male vs. female), age at diagnosis ( $>65$ vs. $<65$ years), Hct during follow-up, platelet count at diagnosis and during followup $\left(<400 \times 10^{9} / 1\right.$ vs. $400-600 \times 10^{9} / 1$ vs. $\left.>600 \times 10^{9} / 1\right)$, white blood cell $(\mathrm{WBC})$ count at diagnosis and during follow-up $\left(<15 \times 10^{9} / 1\right.$ vs. $\left.>15 \times 10^{9} / 1\right)$, thrombotic risk according to ECLAP study (high vs. low-intermediate), prior thrombosis (yes vs. no), cytoreductive treatment (yes vs. no), cardiovascular risk factors at diagnosis (yes vs. no). Since some variables were time-varying, depending on single outcome events (thrombotic risk in OS, Hct, and cytoreductive treatment in TTT and TMT), their impact on OS, TTT, and TMT was then studied by three different multivariate Cox time-dependent models. The cumulative incidence of $\mathrm{AML}, \mathrm{MF}$, and tumors other than AML was determined using the Fine and Gray competing risk regression model. All reported $p$ values are two sided at the conventional 5\% significance level. Data were analyzed as of March 2009 by SPSS 17.0 (SPSS Inc., USA).

\section{Results}

Among the whole casistics of 300 patients, 226 satisfied either 2001 or 2007 WHO diagnostic criteria for PV and underwent further evaluation of clinical outcome.

The main clinical features of the cohort are shown in Table 1. Median follow-up was equal to 5.84 years (range 
$0.76-13.78$ ) and only three patients were lost to follow-up. Known risk factors at diagnosis comprehended history of previous thrombosis in 55 (24\%; arterial thrombosis constituting $85 \%$ and venous thrombosis $15 \%$ of these thrombotic events) and concomitant cardiovascular risk factors in $137(61 \%)$ patients. According to the thrombotic risk stratification, 39 patients were in the low-risk group, 29 in the intermediate one and $158(70 \%)$ in the high-risk group.

All patients received phlebotomy at least in the first month from diagnosis. Seventy-five patients proceeded with phlebotomy only, whereas 151 also received a cytoreductive treatment for at least 6 months (median 4 years). The main cytoreductive treatment was hydroxyurea, used as first-line therapy by 147 (97\%) chemotherapy-treated patients. In particular, HU was the only cytoreductive agent for 126 patients $(83 \%)$, whereas other patients received more than one cytoreductive treatment. Other drugs included pipobroman (19 patients), busulfan (five patients), and alpha interferon (two patients): they were used in the case of intolerance or suboptimal response to HU. Almost all patients (98.2\%) received either anti-platelet (209) and/or anticoagulant (34) therapy.

Median Hct during follow-up was kept at the recommended value below 0.45 in $46 / 211$ evaluable patients (22\%). Sixty-two percent of patients maintained a median Hct value between 0.45 and 0.48 , whereas in $16 \%$ the median Hct value was $>0.48$. Regarding WBC count, a median value above $15 \times 10^{9} / 1$ was observed in 23 patients (11\%). A median platelet value under $400 \times 10^{9} / 1$ was maintained in 110 patients $(52 \%)$, whereas values between $400 \times 10^{9} / 1$ and $600 \times 10^{9} / 1$, or above $600 \times 10^{9} / 1$ were observed in 74 patients $(35 \%)$ and 27 patients $(13 \%)$, respectively.

During follow-up, 25 patients (11\%) died. Survival rate was $91.3 \%$ at the median follow-up of 5.8 years and is projected to $77 \%$ at the longest follow-up of 13.8 years (Fig. 1a). Cardiovascular and thrombotic mortality accounted for $32 \%$ of all deaths, while hematological transformation and solid tumors accounted for $24 \%$ of deaths each. Overall survival was negatively influenced by median Hct over 0.48 ( $p=0.001$; Table 2 and Fig. $1 b$ ) and "high risk" score according to thrombotic risk stratification $(p=0.038)$ (Table 2 and Fig. 1c), whereas sex had no prognostic value (Table 2). At multivariate OS analysis, the impact of median Hct over 0.48 and of high thrombotic risk on total mortality was confirmed, with a hazard ratio (HR) of $3.41(p=0.004)$ and $1.56(p=0.050)$, respectively (Table 3 ). However, due to the low number of dead patients no statistically significant difference was found in the causes of death according to median $\mathrm{Ht}$ value.

Thrombotic events occurred in 58 patients $(25.7 \%)$ during follow-up, after a median time of 58 months (4.8 years). Nineteen percent of patients had a major thrombotic event. The main prognostic factors affecting time to thrombotic events were age over 65 (TTT $p<0.001$, TMT $p=0.001$ ), Hct over 0.48 (TTT $p=0.020$, TMT $p=$ 0.002 ), and high thrombotic risk (TTT $p=0.028$; Table 4, Fig. 2). On the contrary, cytoreductive treatment resulted in a protective agent (TTT $p<0.001$, TMT $p<0.001$ ) (Table 4, Fig. 2). At multivariate analysis, major thrombosis were significantly associated with Hct over $0.48(p=0.011)$ and inversely related to cytoreductive treatment $(p<0.001$; Table 3$)$. Lower Hct values $(\leq 0.45)$ did not significantly discriminate patients in terms of survival and time to thrombosis.

Follow-up WBC and PLT counts did not affect either overall or thrombosis-free survival.

Seventeen and five episodes of major and minor bleeding were observed, respectively. Sixteen events occurred during anti-platelet therapy but only four caused its
Table 2 Prognostic factors for overall survival by univariate Cox model

Values in italics indicate significant variables by univariate analysis

${ }^{\text {a }}$ Time-dependent covariate

\begin{tabular}{|c|c|c|c|}
\hline & HR & $95 \% \mathrm{CI}$ & $P$ value \\
\hline Gender (male vs. female) & 1.46 & $0.65-3.25$ & 0.358 \\
\hline Age at diagnosis $(>65$ vs. $<65$ years) & 10.37 & $3.09-34.75$ & $<0.001$ \\
\hline Hematocrit value $(>0.45$ vs. $<0.45)$ & 2.8 & $0.60-11.01$ & 0.201 \\
\hline Hematocrit value $(>0.48$ vs. $<0.48)$ & 3.90 & $1.69-9.02$ & 0.001 \\
\hline Hematocrit value $(0.45-0.48$ vs. $<0.45)$ & 1.78 & $0.4-7.97$ & 0.45 \\
\hline Hematocrit value $(>0.48$ vs. $<0.45)$ & 6.24 & $1.35-29.9$ & 0.019 \\
\hline Platelets $\left(<400^{\mathrm{a}} 10^{9} / 1\right.$ vs. $400-600^{\mathrm{a}} 10^{9} / 1$ vs. $\left.>600^{\mathrm{a}} 10^{9} / 1\right)$ & 0.61 & $0.32-1.15$ & 0.127 \\
\hline WBC $\left(<12^{\mathrm{a}} 10^{9} / 1\right.$ vs. $\left.>12^{\mathrm{a}} 10^{9} / 1\right)$ & 1.07 & $0.43-2.68$ & 0.886 \\
\hline ECLAP risk (high vs. low-medium) ${ }^{a}$ & 1.61 & $1.03-2.53$ & 0.038 \\
\hline Prior thrombosis (yes vs. no) ${ }^{\mathrm{a}}$ & 1.12 & $0.99-1.27$ & 0.075 \\
\hline Cytoreductive treatment (yes vs. no) & 0.54 & $0.23-1.30$ & 0.170 \\
\hline Cardiovascular risk factors at diagnosis (yes vs. no) & 0.65 & $0.3-1.44$ & 0.289 \\
\hline
\end{tabular}


Table 3 Prognostic factors for overall survival and time to occurrence of thrombotic events by multivariate Cox model

\begin{tabular}{|c|c|c|c|}
\hline & HR & $95 \% \mathrm{CI}$ & $p$ value \\
\hline \multicolumn{4}{|l|}{ Overall survival } \\
\hline Hematocrit value $(>0.48$ vs. $<0.48)$ & 3.41 & $1.47-7.92$ & 0.004 \\
\hline Thrombotic risk (high vs. low-intermediate) ${ }^{a}$ & 1.56 & $1.00-2.44$ & 0.050 \\
\hline \multicolumn{4}{|l|}{ Time to total thrombosis } \\
\hline Hematocrit value $(>0.48 \text { vs. }<0.48)^{\mathrm{a}}$ & 1.11 & $0.98-1.25$ & 0.091 \\
\hline Thrombotic risk (high vs. low-intermediate) & 2.94 & $1.43-6.03$ & 0.003 \\
\hline Cytoreductive treatment (yes vs. no) & 0.32 & $0.18-0.58$ & $<0.001$ \\
\hline \multicolumn{4}{|l|}{ Time to major thrombosis } \\
\hline Hematocrit value $(>0.48 \text { vs. }<0.48)^{a}$ & 1.17 & $1.04-1.31$ & 0.011 \\
\hline Cytoreductive treatment (yes vs. no) & 0.30 & $0.15-0.59$ & $<0.001$ \\
\hline
\end{tabular}

Multivariate analysis tested the following variables that have been demonstrated significative in univariate analysis: Hct value and thrombotic risk (high vs. medium/low) for overall survival; Hct value, thrombotic risk and cytoreductive treatment for time to total thrombosis; Hct value and cytoreductive treatment for time to major thrombosis. Values in italics indicate significant variables

${ }^{\text {a }}$ Time-dependent covariate

interruption. No death occurred as a consequence of hemorrhagic complications.

Leukemic evolution occurred in six patients $(2.7 \%)$, four of whom had received chemotherapy, after a median time of 7 years, with a cumulative incidence of $4.5 \%$ at 10 years. Secondary MF and neoplasia other than AML were observed in $11(4.9 \%)$ and $15(6.6 \%)$ patients, respectively. Evolution to MF occurred after a median time of 7 years and had a cumulative incidence of $7.95 \%$ at 10 years. Chemotherapy did not significantly affect either the hematological transformation or the occurrence of total malignancies (Table 5). Evolution in MF, at univariate analysis, was associated with high Hct levels (over 0.48; $p=0.007$; Table 5).

\section{Discussion}

The present study was aimed to evaluate the clinical management and outcome of PV patients in the past 14 years in our city, outside of clinical trials. In particular, we wished to ascertain, although retrospectively, a possible positive role on survival of the choice to give antithrombotic prophylaxis and to avoid alkylating drugs in

Table 4 Prognostic factors for time to occurrence of thrombotic events by univariate Cox model

\begin{tabular}{|c|c|c|c|c|c|c|}
\hline & \multicolumn{3}{|c|}{ Time to total thrombosis } & \multicolumn{3}{|c|}{ Time to major thrombosis } \\
\hline & HR & $95 \% \mathrm{CI}$ & $p$ value & HR & $95 \% \mathrm{CI}$ & $p$ value \\
\hline Gender (male vs. female) & 1.12 & $0.67-1.89$ & 0.670 & 1.23 & $0.67-2.26$ & 0.5 \\
\hline Age at diagnosis $(>65$ vs. $<65$ yrs $)$ & 2.70 & $1.56-4.68$ & $<0.001$ & 3.07 & $1.61-5.87$ & 0.001 \\
\hline Hematocrit value $(>0.45$ vs. $<0.45)$ & 0.78 & $0.41-1.49$ & 0.454 & 0.97 & $0.45-2.10$ & 0.931 \\
\hline Hematocrit value $(>0.48 \text { vs. }<0.48)^{a}$ & 1.15 & $1.02-1.29$ & 0.020 & 1.20 & $1.07-1.35$ & 0.002 \\
\hline Hematocrit value $(0.45-0.48$ vs. $<0.45)$ & 0.67 & $0.34-1.31$ & 0.244 & 0.75 & $0.33-1.70$ & 0.497 \\
\hline Hematocrit value ( $>0.48 \%$ vs. $<0.45 \%$ ) & 1.30 & $0.9-2.90$ & 0.517 & 2.02 & $0.81-5.02$ & 0.131 \\
\hline WBC $\left(<12^{\mathrm{a}} 10^{9} / 1\right.$ vs. $\left.>12^{\mathrm{a}} 10^{9} / 1\right)$ & 1.09 & $0.60-2.01$ & 0.775 & 1.23 & $0.62-2.46$ & 0.559 \\
\hline ECLAP risk (high vs. low-medium) & 2.09 & $1.08-4.03$ & 0.028 & 1.83 & $0.88-3.81$ & 0.109 \\
\hline Prior thrombosis (yes vs. no) & 1.30 & $0.74-2.26$ & 0.359 & 1.33 & $0.70-2.52$ & 0.382 \\
\hline Cytoreductive treatment (yes vs. no) & 0.34 & $0.20-0.58$ & $<0.001$ & 0.31 & $0.16-0.58$ & $<0.001$ \\
\hline Cardiovascular risk factors at diagnosis (yes vs. no) & 1.01 & $0.59-1.72$ & 0.985 & 0.80 & $0.43-1.48$ & 0.477 \\
\hline
\end{tabular}

Values in italics indicate significant variables by univariate analysis

${ }^{\text {a }}$ Time-dependent covariate 


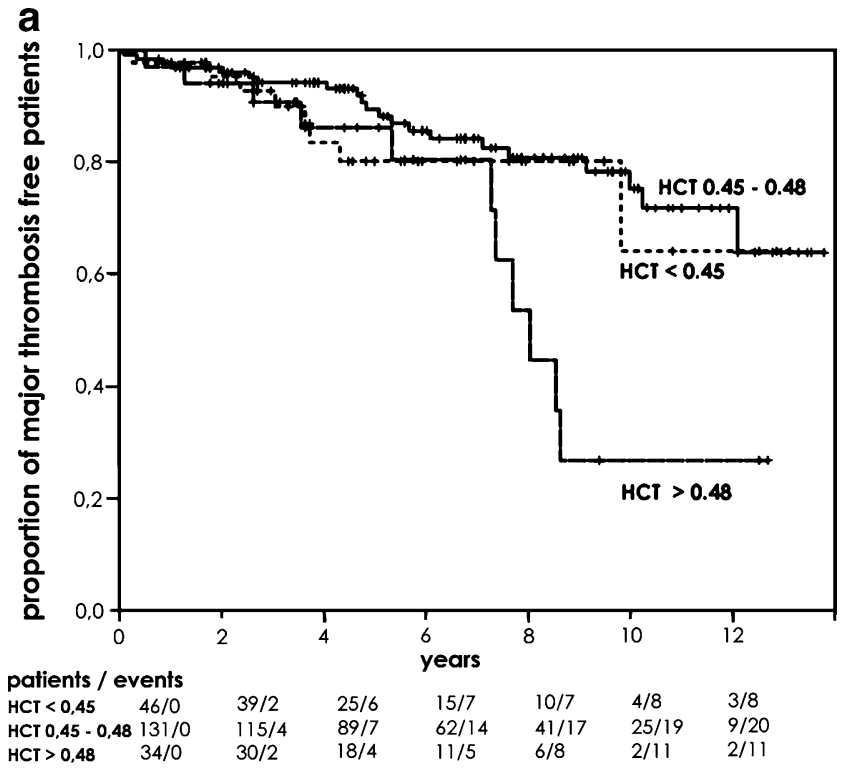

b

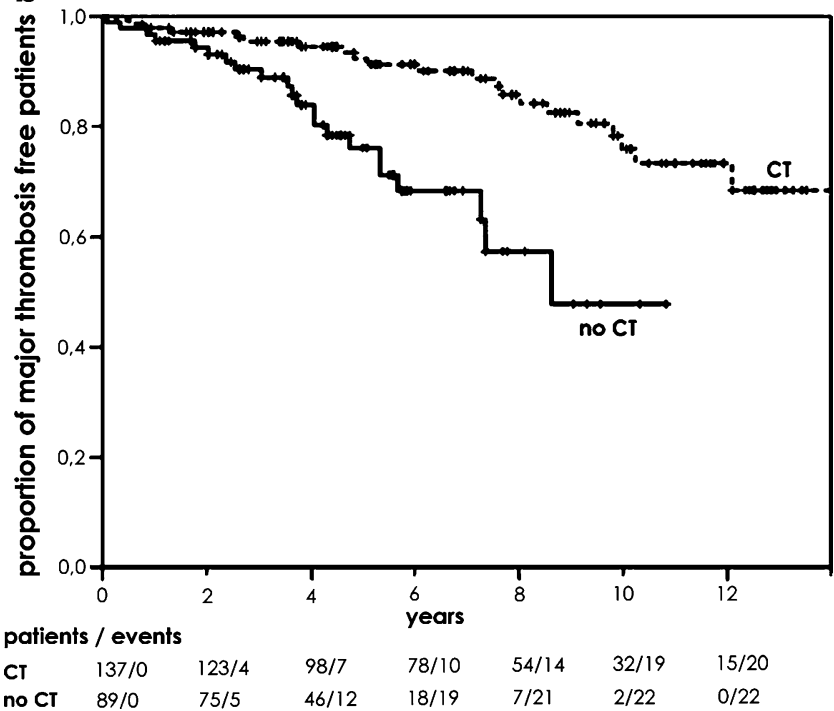

Fig. 2 Major thrombosis-free survival of 226 patients with polycythemia vera. a Major thrombosis-free survival for median hematocrit under 0.45 , between 0.45 and 0.48 and over $0.48(p=0.002)$. b Major thrombosis-free survival for cytoreductive treatment, yes vs. no (HR $0.31, p<0.001)$

$98 \%$ and $89 \%$ of patients, respectively. We also tried to ascertain the clinical impact of median Hct value during the follow-up. Considering the reported PV incidence [1, 2], our casistics probably includes more than $50 \%$ of PV cases occurred during those years in the city of Turin. Follow-up was fairly accurate, as three patients only were lost and, moreover, all evaluated patients had at least three complete blood counts performed/year.

About $25 \%$ of cases referred to our transfusion center for phlebotomy had a PV diagnosis that did not satisfy all WHO diagnostic criteria. Although in many of these cases the diagnosis might have been correct, according to their further clinical outcome, they have been excluded from further evaluations, wishing to restrict prognostic considerations to patients with a doubtless PV diagnosis. In all cases, the unconfirmed diagnosis was made before the analysis of JAK2 status entered the clinical practice. Conversely, $94 \%$ of patients with a PV diagnosis tested in the past 3 years for JAK2 status evidenced the V617F mutation, in agreement to the more recent literature data [25]. These observations illustrate how PV diagnosis might be difficult before the advent of the molecular test and confirm the great impact of JAK2 analysis in improving the diagnostic accuracy.

The median age at diagnosis of our patients (66 years) was relatively advanced, slightly higher than the one previously reported for a large population of unselected Italian PV patients [6]. This certainly contributed to the high proportion $(70 \%)$ of patients in the group of high thrombotic risk. In spite of these unfavorable prognostic features, the overall survival ( $91 \%$ at the median follow-up time of 5.84 years) compares favorably to that reported in many other studies [3-6]. Although we did not perform a prospective, randomized study and the median follow-up is shorter than 10 years, the wide use in our patients of lowdose ASA and the strong limitation of alkylating cytotoxic therapy may have contributed to this more favorable outcome and to an incidence of leukemic transformation in the lowest range of values reported in literature $[4,6-8]$. Indeed, a French randomized study with very long followup has recently demonstrated a lower incidence of leukemic evolution and improved survival with the use of hydroxyurea compared to pipobroman [32].

Low-dose ASA might have also contributed to the relatively low incidence of major thrombosis $(18 \%)$, in the lower range of literature data $[6,27]$, without increasing hemorrhagic complications compared to published observations [33]. Nevertheless, the high thrombotic risk according to Finazzi et al. [28, 29] confirmed its negative prognostic impact in terms of overall and thrombosis-free survival in both univariate and multivariate analysis.

Differently from what is reported in another study [34], WBC value during follow-up did not significantly affect survival and thrombotic complications. However, few patients $(22: 10.2 \%)$ maintained a value above the reported threshold of $15 \times 10^{9} / 1$ : this may explain the lack of significant difference in thrombosis-free survival. Chemotherapy given before the occurrence of a thrombotic event significantly prolonged the freedom from major thrombosis but did not affect overall survival, in agreement to some previous reports $[14,16,28]$.

Regarding Hct target, the need of keeping a value $<0.45$, as recommended by guidelines [18, 29, 35] has been recently questioned by the ECLAP study [30]. Unfortu- 
Table 5 Prognostic factors for occurrence of acute myeloid leukemia. Solid tumors or myelofibrosis by univariate Cox model

\begin{tabular}{|c|c|c|c|c|c|c|}
\hline & \multicolumn{3}{|c|}{ Time to AML or solid tumors } & \multicolumn{3}{|c|}{ Time to myelofibrosis } \\
\hline & HR & $95 \% \mathrm{CI}$ & $p$ value & HR & $95 \% \mathrm{CI}$ & $p$ value \\
\hline Gender (male vs. female) & 2.17 & $0.86-5.46$ & 0.101 & 0.99 & $0.29-3.43$ & 0.989 \\
\hline Age at diagnosis ( $>65$ vs. $<65$ yrs) & 1.69 & $0.71-4.04$ & 0.237 & 1.03 & $0.29-3.69$ & 0.961 \\
\hline Hematocrit value $(>0.45$ vs. $<0.45)$ & 0.57 & $0.22-1.49$ & 0.254 & 0.92 & $0.19-4.33$ & 0.913 \\
\hline Hematocrit value $(>0.48$ vs. $<0.48)$ & 0.72 & $0.17-3.14$ & 0.666 & $1.25^{\mathrm{a}}$ & $1.06^{\mathrm{a}}-1.47^{\mathrm{a}}$ & $0.007^{\mathrm{a}}$ \\
\hline Hematocrit value $(0.45-0.48$ vs. $<0.45)$ & 0.59 & $0.22-1.57$ & 0.290 & 0.52 & $0.10-2.85$ & 0.452 \\
\hline Hematocrit value $(>0.48$ vs. $<0.45)$ & 0.50 & $0.10-2.46$ & 0.391 & 3.53 & $0.64-19.63$ & 0.149 \\
\hline Platelets $\left(<400^{\mathrm{a}} 10^{9} / 1\right.$ vs. $400-600^{\mathrm{a}} 10^{9} / 1$ vs. $\left.>600^{\mathrm{a}} 10^{9} / 1\right)$ & 1.33 & $0.70-2.50$ & 0.382 & 0.84 & $0.35-2.01$ & 0.690 \\
\hline WBC $\left(<12^{\mathrm{a}} 10^{9} / 1\right.$ vs. $\left.>12^{\mathrm{a}} 10^{9} / 1\right)$ & 0.19 & $0.03-1.46$ & 0.111 & 0.82 & $0.17-3.86$ & 0.801 \\
\hline ECLAP risk (high vs. low-medium) & $1.19^{\mathrm{a}}$ & $0.46^{\mathrm{a}}-3.08^{\mathrm{a}}$ & $0.715^{\mathrm{a}}$ & 1.93 & $0.41-9.11$ & 0.404 \\
\hline Cytoreductive treatment (yes vs. no) & 0.40 & $0.16-1.01$ & 0.053 & 1.79 & $0.22-14.87$ & 0.590 \\
\hline
\end{tabular}

Values in italics indicate significant variables by univariate analysis

${ }^{\text {a }}$ Time-dependent covariate

nately, few patients ( $22 \%$ only) in our survey were actually maintained at an Hct value $<0.45$, this suggesting the difficulties for patients and/or physicians to comply with published guidelines, in agreement to other reports [30,36]. Although this group of patients had a favorable outcome, no significant differences could be detected in terms of overall or thrombosis-free survival compared to the larger group of patients who maintained median Hct values in the range of $0.45-0.48$. Conversely, the few patients $(16 \%)$ with a poor control of erythrocytosis (median Hct value> 0.48 ) had a significantly worst outcome in terms of both overall survival and time to major thrombosis, both by univariate and multivariate analysis.

In conclusion, although our study is retrospective and the casistic is not very large, the results obtained on unselected PV patients receiving routine care are in agreement to the already reported positive roles of antiplatelet therapy and of hydroxyurea as a cytoreductive drug. The role of JAK2 status analysis in improving the diagnostic accuracy has been also confirmed, as well as the negative impact of belonging to the high thrombotic risk category. Other known risk factors, such as leukocytosis and prior thrombosis could not be confirmed in our casistic, probably because of the relatively limited number of patients. Regarding one of the most controversial point in the last years, the Hct value to be pursued, we believe on the basis of our results that a good control of erythrocytosis, with an Hct value below 0.48 may be advisable, although definitive conclusions on the optimal value have not been reached. A randomized study is ongoing in Italy comparing different Hct targets and hopefully should provide more sound information about the optimal value to be achieved.
Acknowledgment This study was in part supported by the Università degli Studi di Torino (ex 60\% grants)

\section{References}

1. Silverstein MN, Lanier AP (1971) Polycythemia vera, 19351969: an epidemiologic survey in Rochester, Minnesota. Mayo Clin Proc 46:751-753

2. Ania BJ, Suman VJ, Sobell JL, Codd MB, Silverstein MN, Melton LJ 3rd (1994) Trends in the incidence of polycythemia vera among Olmested Country, Minnesota residents, 1935-1989. Am J Hematol 47:89-93

3. Cervantes F, Passamonti F, Barosi G (2008) Life expectancy and prognostic factors in the classic BCR/ABL-negative myeloproliferative disorders. Leukemia 22:905-914, Epub 2008 Apr 3

4. Kiladjian JJ, Gardin C, Renoux M, Bruno F, Bernard JF (2003) Long-term outcomes of polycythemia vera patients treated with pipobroman as initial therapy. Hematol J 4:198-207

5. Finazzi G, Barbui T (2007) The treatment of polycythaemia vera: an update in the JAK2 era. Intern Emerg Med 2:13-18, Epub 2007 Mar 31

6. Gruppo Italiano Studio Policitemia (1995) Polycythemia vera: the natural history of 1213 patients followed for 20 years. Ann Intern Med 123:656-664

7. Ellis JT, Peterson P, Geller SA, Rappaport H (1986) Studies of the bone marrow in polycythemia vera and the evolution of myelofibrosis and second hematologic malignancies. Semin Hematol 23:144-155

8. Silverstein MN (1976) The evolution into and the treatment of late stage polycythemia vera. Semin Hematol 13:79-84

9. Finazzi G, Caruso V, Marchioli R, Capnist G, Chisesi T, Finelli C, Gugliotta L, Landolfi R, Kutti J, Gisslinger H, Marilus R, Patrono C, Pogliani EM, Randi ML, Villegas A, Tognoni G, Barbui T, ECLAP Investigators (2005a) Acute leukemia in polycythemia vera: an analysis of 1638 patients enrolled in a prospective observational study. Blood 105:2664-2670, Epub 2004 Dec 7

10. Berk PD, Goldberg JD, Silverstein MN, Weinfeld A, Donovan PB, Ellis JT, Landaw SA, Laszlo J, Najean Y, Pisciotta AV, Wasserman LR (1981) Increased incidence of acute leukemia in 
polycythemia vera associated with chlorambucil therapy. N Engl J Med 304:441-447

11. Cooperative Group, European Organization for Research on Treatment of Cancer (E.O.R.T.C.) (1981) Treatment of polycythaemia vera by radiophosphorus or busulphan: a randomized trial. "Leukemia and Hematosarcoma". Br J Cancer 44:75-80

12. Messinezy M, Pearson TC, Prochazka A, Wetherley-Mein G (1985) Treatment of primary proliferative polycythaemia by venesection and low dose busulphan: retrospective study from one centre. Br J Haematol 61:657-666

13. Najean Y, Rain JD, for the French Polycythemia Study Group (1997) Treatment of polycythemia vera: use of ${ }^{32} \mathrm{P}$ alone or in combination with maintenance therapy using hydroxyurea in 461 patients greater than 65 years of age. Blood 89:2319-2327

14. Najean Y, Rain JD, for the French Polycythemia Study Group (1997) Treatment of polycythemia vera: the use of hydroxyurea and pipobroman in 292 patients under the age of 65 years. Blood 90:3370-3377

15. Najean Y, Rain JD (1997) A very long-term evolution of polycythemia vera: an analysis of 318 patients initially treated by phlebotomy or ${ }^{32} \mathrm{P}$ between 1969 and 1981. Semin Hematol 34:6-16

16. Fruchtman SM, Mack K, Kaplan ME, Peterson P, Berk PD, Wasserman LR (1997) From efficacy to safety: a Polycythemia Vera Study Group report on hydroxyurea in patients with polycythemia vera. Semin Hematol 34:17-23

17. Wasserman LR (1986) Polycythemia Vera Study Group: a historical perspective. Semin Hematol 23:183-187

18. McMullin MF, Bareford D, Campbell P, Green AR, Harrison C, Hunt B, Oscier D, Polkey MI, Reilly JT, Rosenthal E, Ryan K, Pearson TC, Wilkins B, General Haematology Task Force of the British Committee for Standards in Haematology (2005) Guidelines for the diagnosis, investigation and management of polycythaemia /erythrocytosis. Br J Haematol 130:174-195

19. Tefferi A, Thiele J, Orazi A, Kvasnicka HM, Barbui T, Hanson CA, Barosi G, Verstovsek S, Birgegard G, Mesa R, Reilly JT, Gisslinger H, Vannucchi AM, Cervantes F, Finazzi G, Hoffman R, Gilliland DG, Bloomfield CD, Vardiman JW (2007) Proposals and rationale for revision of the World Health Organization diagnostic criteria for polycythemia vera, essential thrombocythemia, and primary myelofibrosis: recommendations from an ad hoc international expert panel. Blood 110:1092-1097, Epub 2007 May 8

20. Spivak JL, Silver RT (2008) The revised World Health Organization diagnostic criteria for polycythemia vera, essential thrombocytosis, and primary myelofibrosis: an alternative proposal. Blood 112:231-239, Epub 2008 Apr 9

21. Levine RL, Wadleigh M, Cools J, Ebert BL, Wernig G, Huntly BJ, Boggon TJ, Wlodarska I, Clark JJ, Moore S, Adelsperger J, Koo S, Lee JC, Gabriel S, Mercher T, D'Andrea A, Fröhling S, Döhner K, Marynen P, Vandenberghe P, Mesa RA, Tefferi A, Griffin JD, Eck MJ, Sellers WR, Meyerson M, Golub TR, Lee SJ, Gilliland DG (2005) Activating mutation in the tyrosine kinase JAK2 in polycythemia vera, essential thrombocythemia, and myeloid metaplasia with myelofibrosis. Cancer Cell 7:387397

22. Baxter EJ, Scott LM, Campbell PJ, East C, Fourouclas N, Swanton S, Vassiliou GS, Bench AJ, Boyd EM, Curtin N, Scott MA, Erber WN, Green AR, Cancer Genome Project (2005) Acquired mutation of the tyrosine kinase JAK2 in human myeloproliferative disorders. Lancet 365:1054-1061, Erratum in: Lancet 2005;366:122

23. Kralovics R, Passamonti F, Buser AS, Teo SS, Tiedt R, Passweg JR, Tichelli A, Cazzola M, Skoda RC (2005) A gain-of-function mutation of JAK2 in myeloproliferative disorders. N Engl J Med 352:1779-1790

24. Scott LM, Beer PA, Bench AJ, Erber WN, Green AR (2007) Prevalence of JAK2 V617F and exon 12 mutations in polycythaemia vera. Br J Haematol 139:511-512

25. Skoda R (2007) The genetic basis of myeloproliferative disorders. Hematology, the Education Program of the American Society of Hematology 2007:1-10

26. McMullin MF (2007) A review of the therapeutic agents used in the management of polycythaemia vera. Hematol Oncol 25:58-65

27. Landolfi R, Marchioli R, Kutti J, Gisslinger H, Tognoni G, Patrono C, Barbui T, European Collaboration on Low-Dose Aspirin in Polycythemia Vera Investigators (2004) Efficacy and safety of low-dose aspirin in polycythemia vera. N Engl J Med 350:114-124

28. Finazzi G, Barbui T (2005) Risk-adapted therapy in essential thrombocythemia and polycythemia vera. Blood Rev 19:243-252

29. Finazzi G, Barbui $T$ (2008) Evidence and expertise in the management of polycythemia vera and essential thrombocythemia. Leukemia 22:1494-1502, Epub 2008 Jul 3

30. Di Nisio M, Barbui T, Di Gennaro L, Borrelli G, Finazzi G, Landolfi R, Leone G, Marfisi R, Porreca E, Ruggeri M, Rutjes AW, Tognoni G, Vannucchi AM, Marchioli R, European Collaboration on Lowdose Aspirin in Polycythemia Vera (ECLAP) Investigators (2007) The haematocrit and platelet target in polycythemia vera. $\mathrm{Br} \mathrm{J}$ Haematol 136:249-259, Epub 2006 Dec 8

31. Vardiman JW, Brunning RD, Harris NL (2001) WHO histological classification of chronic myeloproliferative diseases. In: Jaffe ES, Harris NL, Stein H, Vardiman JW (eds) World Health Organization Classification of Tumors: Tumours of the Haematopoietic and Lymphoid Tissues. International Agency for Research on Cancer (IARC) Press, Lyon, France, pp 17-44

32. Kiladjian JJ, Chevret S, Dosquet C, Fenaux P, Chomienne C, Rain JD (2008) Long-Term outcome in Polycythemia Vera (PV): final analysis of a randomized trial comparing hydroxyurea (HU) to pipobroman (Pi). Blood (ASH Annual Meeting Abstracts) 112:1746

33. Marchioli R, Finazzi G, Landolfi R, Kutti J, Gisslinger H, Patrono C, Marilus R, Villegas A, Tognoni G, Barbui T (2005) Vascular and neoplastic risk in a large cohort of patients with polycythemia vera. J Clin Oncol 23:2224-2232, Epub 2005 Feb 14

34. Landolfi R, Di Gennaro L, Barbui T, De Stefano V, Finazzi G, Marfisi R, Tognoni G, Marchioli R, European Collaboration on Low-Dose Aspirin in Polycythemia Vera (ECLAP) (2007) Leukocytosis as a major thrombotic risk factor in patients with polycythemia vera. Blood 109:2446-2452, Epub 2006 Nov 14

35. Vannucchi AM, Guglielmelli P, Tefferi A (2009) Advances in understanding and management of myeloproliferative neoplasms. CA Cancer J Clin 57:171-191, Apr 15

36. Streiff MB, Smith B, Spivak JL (2002) The diagnosis and management of polycythemia vera in the era since the Polycythemia Vera Study Group: a survey of American Society of Hematology members' practice patterns. Blood 99:1144-1149 